\title{
Role of Water Hydrogen Bonding on Transport of Small Molecules Inside Hydrophobic Channels
}

\author{
Diego E. Escalante, ${ }^{\dagger}$ and Alptekin Aksan $\uparrow^{\dagger} *$ \\ $\dagger$ Department of Mechanical Engineering, University of Minnesota, Minneapolis, MN 55455, \\ United States. \\ §BioTechnology Institute, University of Minnesota, St. Paul, MN 55108, United States. \\ *Corresponding author. Email: aaksan@umn.edu
}

\section{$\underline{\text { Supporting information content }}$}

§SI 1. Construction of Building Block Surfaces

§SI 2. Calculation of Water Angular Distribution Angles

§SI 3. Ligand Diffusion Coefficients

§SI 4. Supporting Graphs

§SI 5. Analysis of building block scope for modeling real enzyme channels

§SI 6. References 


\section{§SI 1. Construction of Building Block Surfaces}

The surface of a hyperboloid is given in Cartesian coordinates by Equation S1:

$$
\frac{x^{2}}{a^{2}}+\frac{y^{2}}{b^{2}}-\frac{z^{2}}{c^{2}}=1
$$

where $a^{2}=b^{2}=r_{o}^{2}$ for a radially symmetrical channel as it is in our case. Equivalently, Equation

$\boldsymbol{S 1}$ can be represented in cylindrical coordinated by Equation $\boldsymbol{S} 2$ :

$$
\frac{r^{2}}{r_{o}^{2}}-\frac{z^{2}}{c^{2}}=1
$$

and the geometry constant $c$ is given by Equation S3:

$$
c=\frac{r_{o} L}{\sqrt{\left(r_{i}+r_{o}\right)\left(r_{i}-r_{o}\right)}}
$$

In order to obtain building blocks with wall thickness of two atoms we deleted all the atoms where $r<5$ and $r>7$. 


\section{§SI 2. Calculation of Water Angular Distribution Angles}

The angles $\theta$ and $\chi$ are used to describe the rotational orientation of each water molecule. The definition of each angle is as follow:

$$
\begin{aligned}
& \theta=\cos ^{-1}\left(\frac{r_{\mathrm{lw}} \cdot \mu}{\left|r_{\mathrm{lw}} \cdot \mu\right|}\right) \\
& \chi=\cos ^{-1}\left(\frac{h \cdot n}{|h \cdot n|}\right)
\end{aligned}
$$

Vector $r_{\mathrm{lw}}$ is the distance from the oxygen $\left(\bar{x}_{\text {oxygen }}\right)$ atom to the origin:

$$
r_{\mathrm{lw}}=\left|\bar{x}_{\text {oxygen }}\right|
$$

Vector $h$ is the direction of from hydrogen $1\left(\bar{x}_{\text {hydrogen - } 1}\right)$ to hydrogen $2\left(\bar{x}_{\text {hydrogen - }}\right)$

$$
h=\bar{x}_{\text {hydrogen - } 2}-\bar{x}_{\text {hydrogen }-1}
$$

Vector $\mu$ is the direction of the water molecule dipole moment:

$$
\mu=\left(-0.82 \times \bar{x}_{\text {oxygen }}\right)+\left(0.41 \times \bar{x}_{\text {hydrogen - } 1}\right)+\left(0.41 \times \bar{x}_{\text {hydrogen - } 2}\right)
$$

Vector $n$ is perpendicular to $r_{\mathrm{lw}}$ and $\mu$

$$
n=r_{\mathrm{lw}} \times \mu
$$




\section{§SI 3. Ligand Diffusion Coefficients}

Diffusion coefficients for individual direction components of ligand were calculated and shown in Table SI 1

Table SI 1. Diffusion coefficients for ligands inside BB geometries: (top) cylinder, (middle) barrel, and (bottom) hourglass. The radial $\left(D_{\mathrm{R}}\right)$ and axial $\left(D_{\mathrm{Z}}\right)$ diffusion coefficients are in $\mathrm{cm}^{2} / \mathrm{s}$, and the tangential $\left(D_{\theta}\right)$ diffusion coefficients is in $\mathrm{deg}^{2} / \mathrm{s}$.

\begin{tabular}{|c|c|c|c|}
\hline$\varepsilon_{\mathrm{W}} / \varepsilon_{\mathrm{L}}$ & $\begin{array}{c}\mathbf{D}_{\mathbf{R}} \\
\left(\times 10^{5}\right)\end{array}$ & $\begin{array}{c}\mathbf{D}_{\mathbf{Z}} \\
\left(\times 10^{5}\right)\end{array}$ & $\mathbf{D}_{\theta}$ \\
\hline 0.5 & 6.29 & 13.86 & 31 \\
\hline 0.6 & 5.83 & 13.51 & 29 \\
\hline 0.7 & 5.17 & 11.62 & 28 \\
\hline 0.8 & 1.56 & 2.90 & 18 \\
\hline 0.9 & 1.29 & 2.41 & 17 \\
\hline 1.0 & 1.25 & 2.31 & 16 \\
\hline$\varepsilon_{\mathrm{W}} / \boldsymbol{\varepsilon}_{\mathrm{L}}$ & $\begin{array}{c}\mathbf{D}_{\mathbf{R}} \\
\left(\times 10^{5}\right)\end{array}$ & $\begin{array}{c}\mathbf{D}_{\mathbf{Z}} \\
\left(\times 10^{5}\right)\end{array}$ & $\mathbf{D}_{\theta}$ \\
\hline 0.5 & 7.08 & 18.12 & 30 \\
\hline 0.6 & 6.75 & 18.08 & 29 \\
\hline 0.7 & 6.44 & 16.44 & 28 \\
\hline 0.8 & 6.09 & 16.01 & 26 \\
\hline 0.9 & 3.36 & 8.31 & 24 \\
\hline 1.0 & 2.89 & 7.15 & 20 \\
\hline$\varepsilon_{\mathrm{W}} / \varepsilon_{\mathrm{L}}$ & $\begin{array}{c}\mathbf{D}_{\mathbf{R}} \\
\left(\times 10^{5}\right)\end{array}$ & $\begin{array}{c}\mathbf{D}_{\mathbf{Z}} \\
\left(\times 10^{5}\right)\end{array}$ & $\mathbf{D}_{\theta}$ \\
\hline 0.5 & 3.94 & 8.80 & 30 \\
\hline 0.6 & 3.84 & 8.60 & 64 \\
\hline 0.7 & 3.61 & 8.01 & 15 \\
\hline 0.8 & 3.49 & 7.92 & 14 \\
\hline 0.9 & 3.46 & 7.86 & 13 \\
\hline 1.0 & 3.49 & 7.81 & 12 \\
\hline
\end{tabular}




\section{§SI 4. Supporting Graphs}
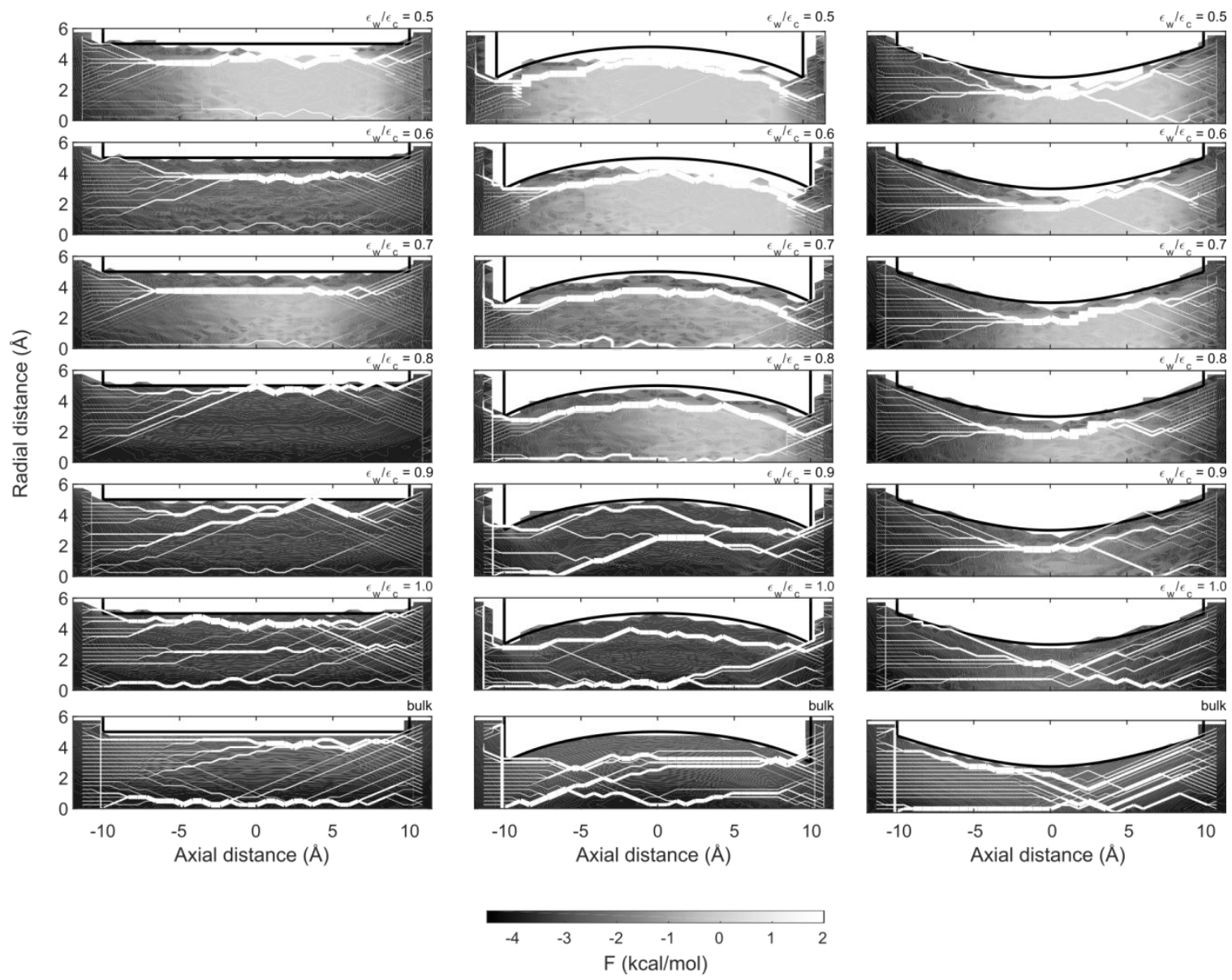

Supplemental Figure SI 1. Potential Energy Surface (PES) maps showing the energetic barrier needed to transport a ligand along the axial and radial. Left column are cylinder BB, middle column are barrel BB and right column are hourglass BB. Bulk water paths are shown in the last row for all three types of BB. The white lines show the different paths taken by the ligands while transported downstream $(-12 \AA<\mathrm{z}<$ $12 \AA)$. Thicker lines indicate a higher probability that a ligand would flow down that path. 

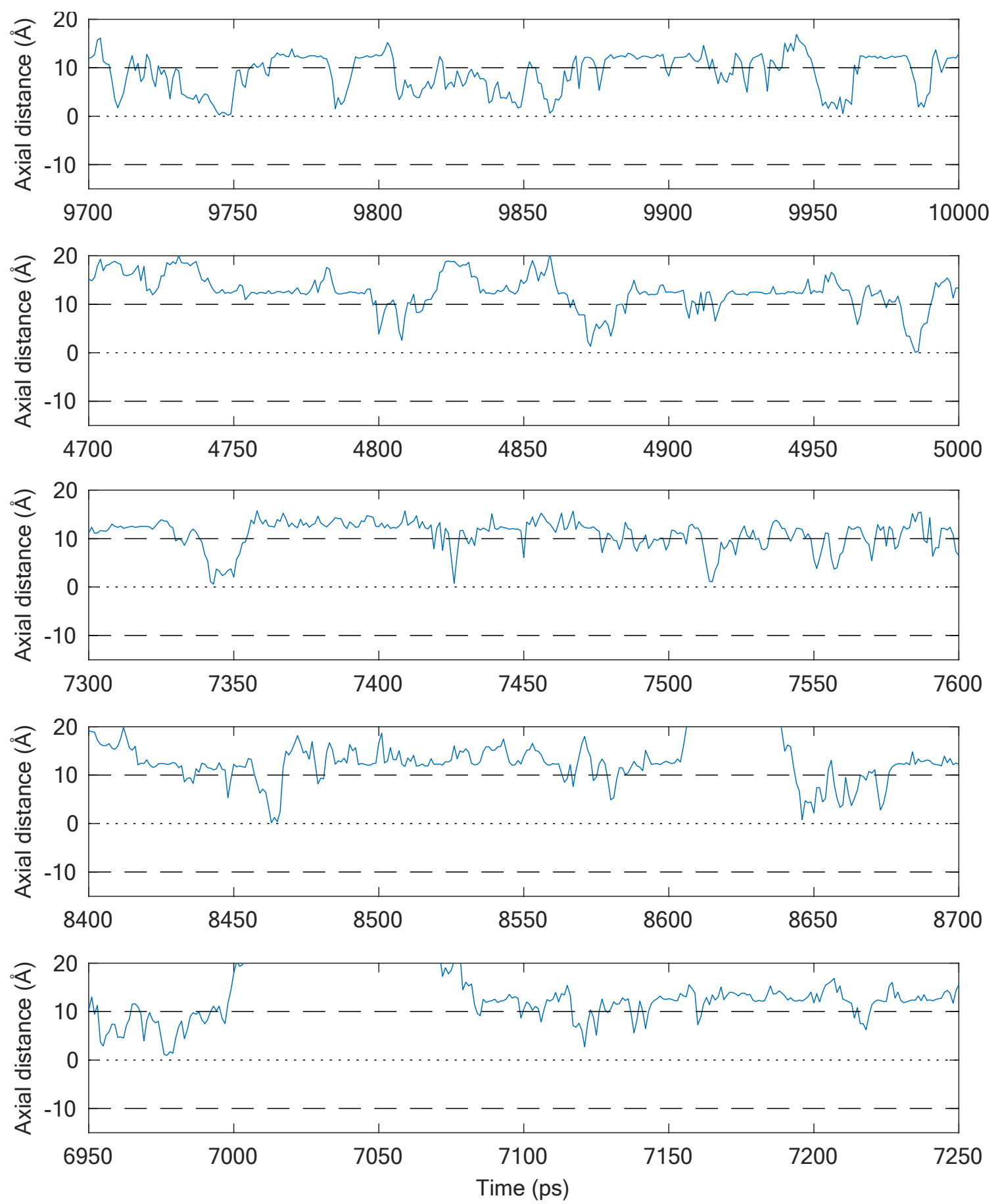

Supplemental Figure SI 2. Snapshots showing the location of different ligands along the axial direction in the hourglass BB. The bottleneck is at $\mathrm{z}=0 \AA$ shown as the dotted line, and the dashed lines show the entrance and exit regions. 


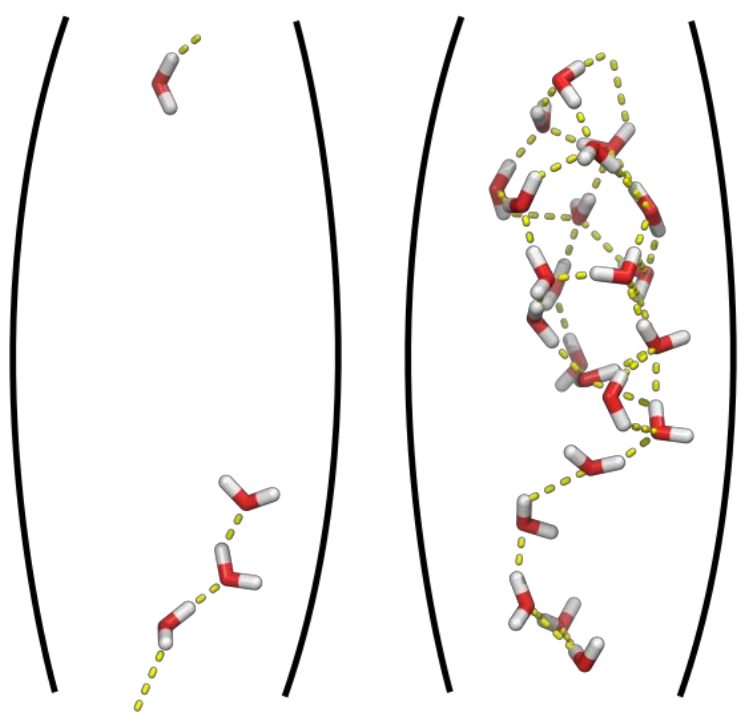

Supplemental Figure SI 3. Snapshot showing distribution of water molecules inside the barrel BB at $\mathrm{PWR}=0.5$ (left) and $\mathrm{PWR}=0.8$ (right). 


\section{§SI 5. Analysis of building block scope for modeling real enzyme channels}

We now discuss their scope of our building blocks as coarse-grain models of real enzyme channels. We acknowledge that our BBs are not applicable to every enzyme channel, but rather to a subset that fulfills the conditions of hydrophobicity and low polarity. In order to determine a set of possible enzymes that fulfills these conditions we analyzed the supplementary data provided by Pravda et. al. ${ }^{1}$ In this list they provide average physicochemical properties of over 8000 different channels covering the entire spectrum of enzyme as classified by the enzyme commission (EC) number. The EC classification is a hierarchical scheme in which enzymes are grouped together based on the reaction they catalyze. ${ }^{2} \mathrm{EC}$ classes are expressed as string of four numbers separated by periods (i.e. 1.14.12.12 the EC number for naphthalene 1,2-dioxygenase), and the second digit (i.e. the subclass) contains information about the type of compound involved. Table SI 2 shows the eight subclasses of EC numbers that fit the defined criteria. In addition, we have outlined the total number of crystal structures available as well as the total number of organisms within each respective EC subclass. The full list of EC numbers and their channel average hydrophobicity value can be found in Figure SI 4. Since hydrophobicity and polarity are negatively correlated (Figure SI 5) we are confident that ignoring possible hydrogen bonds in our BBs is a valid assumption.

Table SI 2. Subclasses of enzymes containing hydrophobic channels leading into the active site.

\begin{tabular}{|l|l|c|c|}
\hline $\begin{array}{l}\text { E.C } \\
\text { Subclass }\end{array}$ & Description & $\begin{array}{c}\text { PDB } \\
\text { Entries }\end{array}$ & $\begin{array}{c}\text { Number of } \\
\text { Organisms }\end{array}$ \\
\hline 1.9 & Acting on a heme group of donors & 557 & 340 \\
\hline 1.12 & Acting on hydrogen as donor & 268 & 259 \\
\hline 1.13 & $\begin{array}{l}\text { Acting on single donors with incorporation of } \\
\text { molecular oxygen (oxygenases) }\end{array}$ & 640 & 850 \\
\hline 1.14 & $\begin{array}{l}\text { Acting on paired donors, with incorporation or } \\
\text { reduction of molecular oxygen }\end{array}$ & 3200 & 2346 \\
\hline 1.21 & Acting on X-H and Y-H to form an X-Y bond & 65 & 146 \\
\hline 3.7 & Acting on carbon-carbon bonds & 71 & 140 \\
\hline 3.8 & Acting on halide bonds & 166 & 157 \\
\hline 6.4 & Forming carbon-carbon bonds & 122 & 202 \\
\hline
\end{tabular}

Based on the results shown in Table SI 2 we have identified at least eight subclasses of enzymes for which the BBs can be used to study the transport of ligands into their buried active sites, yielding a total of $>3500$ enzymes. Furthermore, there are enzymes channels belonging to other EC subclasses for which the hydrophobicity and low polarity conditions could also be met, however, those would need to be identified on a case-by-case basis. For the eight identified EC subclasses, the majority of the channels found have been shown to be highly hydrophobic, in particular those belonging to of EC 1.9. and EC 1.13. The enzymes belonging to these two subclasses can be broadly classified as promiscuous enzymes, meaning that they are capable of accepting a myriad of substrates, ${ }^{3}$ in some cases allowing the access of hundreds of chemical compounds into their buried active sites. ${ }^{4-11}$ Therefore, although the scope of our BBs is confined to a narrow type of enzyme channels the total number of possible organisms with enzymes exhibiting hydrophobic nanochannels is well into the thousands. And when coupled with the inherent promiscuity of these enzymes, the range of organic compounds that can be obtained from biocatalysis can reach hundreds of thousands of possibilities. 


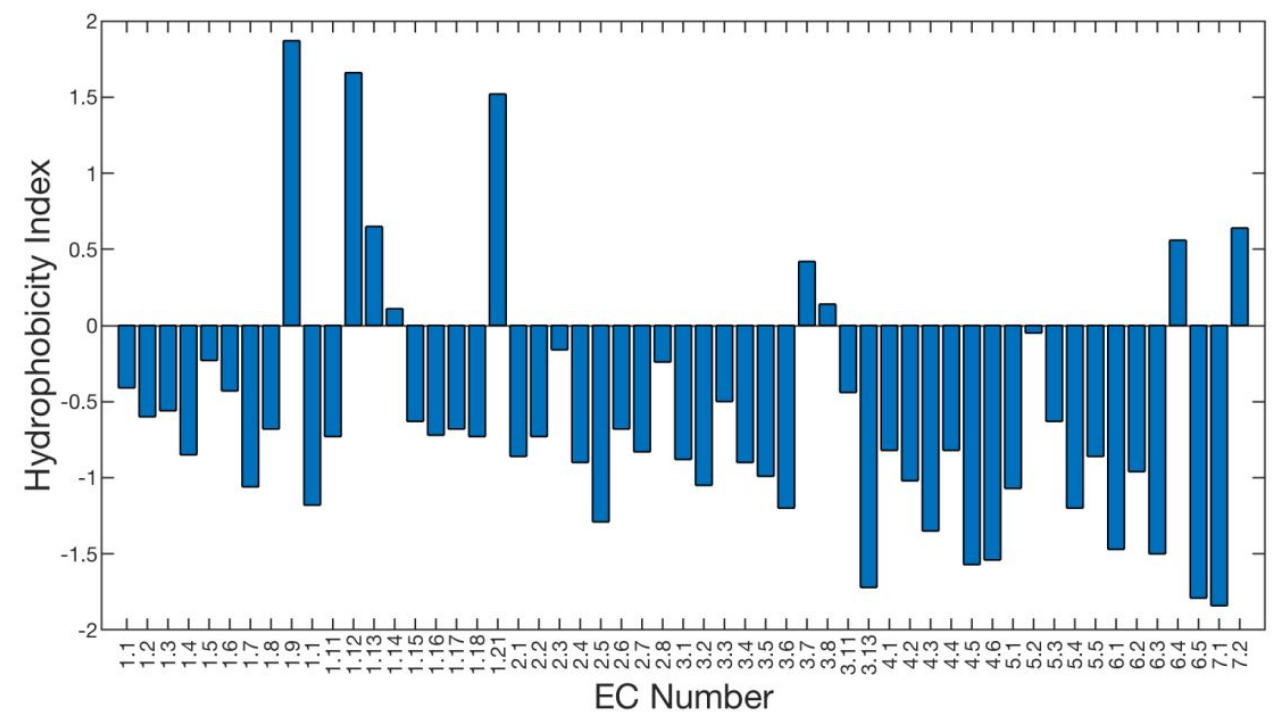

Supplemental Figure SI 4. Hydrophobicity index of channels found in enzymes belonging to each enzyme commission (EC) subclass. A positive value indicates the presence of primarily hydrophobic residues inside the channel, whereas a negative value indicates the presence of primarily hydrophilic residues inside the channel.

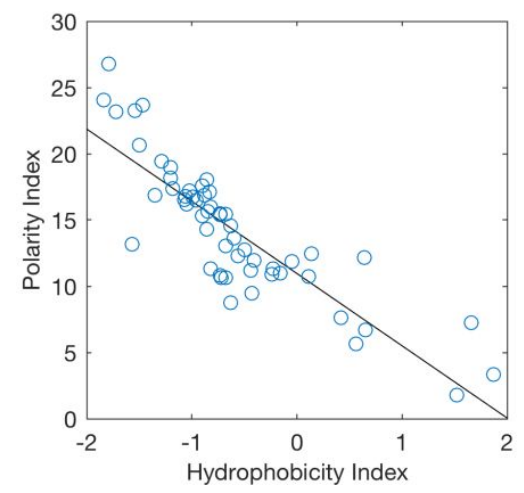

Supplemental Figure SI 5 Relationship between polarity index and hydrophobicity index for residues found inside channels for enzymes belonging to all EC subclasses. A hydrophobic index positive value indicates the presence of primarily hydrophobic residues inside the channel, whereas a negative value indicates the presence of primarily hydrophilic residues inside the channel. Lower polarity index values indicate the absence of polar residues, i.e. less chances of forming hydrogen bonds with water or ligands traversing the channel. 


\section{§SI 6. References}

1. Pravda, L.; Berka, K.; Svobodova Varekova, R.; Sehnal, D.; Banas, P.; Laskowski, R. A.; Koca, J.; Otyepka, M., Anatomy of Enzyme Channels. BMC Bioinformatics 2014, 15, 379.

2. McDonald, A. G.; Tipton, K. F., Fifty-Five Years of Enzyme Classification: Advances and Difficulties. Febs $J$ 2014, 281, 583-592.

3. Copley, S. D., Enzymes with Extra Talents: Moonlighting Functions and Catalytic Promiscuity. Curr Opin Chem Biol 2003, 7, 265-272.

4. $\quad$ Aukema, K. G.; Kasinkas, L.; Aksan, A.; Wackett, L. P., Use of Silica Encapsulated Pseudomonas Sp. Strain Ncib 9816-4 in Biodegradation of Novel Hydrocarbon Ring Structures Found in Hydraulic Fracturing Waters. Appl. Environ. Microbiol. 2014, 80, 4968-4976.

5. $\quad$ Cheng, F. X.; Yu, Y.; Shen, J.; Yang, L.; Li, W. H.; Liu, G. X.; Lee, P. W.; Tang, Y., Classification of Cytochrome P450 Inhibitors and Noninhibitors Using Combined Classifiers. J. Chem. Inf. Model. 2011, 51, 996-1011.

6. Cui, Y. L.; Zhang, J. L.; Zheng, Q. C.; Niu, R. J.; Xu, Y.; Zhang, H. X.; Sun, C. C., Structural and Dynamic Basis of Human Cytochrome P450 7b1: A Survey of Substrate Selectivity and Major Active Site Access Channels. Chemistry 2013, 19, 549-557.

7. Fishelovitch, D.; Shaik, S.; Wolfson, H. J.; Nussinov, R., Theoretical Characterization of Substrate Access/Exit Channels in the Human Cytochrome P450 3a4 Enzyme: Involvement of Phenylalanine Residues in the Gating Mechanism. J. Phys. Chem. B 2009, 113, 13018-13025.

8. Guengerich, F. P., Cytochrome P450 and Chemical Toxicology. Chem. Res. Toxicol. 2008, 21, $70-83$.

9. Jung, S. T.; Lauchli, R.; Arnold, F. H., Cytochrome P450: Taming a Wild Type Enzyme. Curr Opin Biotech 2011, 22, 809-817.

10. Ludemann, S. K.; Lounnas, V.; Wade, R. C., How Do Substrates Enter and Products Exit the Buried Active Site of Cytochrome P450cam? 2. Steered Molecular Dynamics and Adiabatic Mapping of Substrate Pathways. J. Mol. Biol. 2000, 303, 813-830.

11. Olsen, L.; Oostenbrink, C.; Jorgensen, F. S., Prediction of Cytochrome P450 Mediated Metabolism. Adv. Drug. Deliver. Rev. 2015, 86, 61-71. 